\title{
Cardiac Severity Analysis
}

Fatima Mokeddem ${ }^{1}$ and Sidi Mohamed Debbal ${ }^{1 *}$

${ }^{1}$ Genie-Biomedical Laboratory (GBM), Faculty of Technology, University of Aboubekr Belkaid - Tlemcen, BP 119, Tlemcen (Algeria).

*Corresponding Author: Sidi Mohamed Debbal, Genie-Biomedical Laboratory (GBM), Faculty of Technology, University of Aboubekr Belkaid - Tlemcen, BP 119, Tlemcen (Algeria).

Received date: May 28, 2021; Accepted date: July 30, 2021; Published date: August 11, 2021

Citation: Fatima Mokeddem, Sidi M. Debbal (2021). Cardiac Severity Analysis. J Thoracic Disease and Cardiothoracic Surgery, 2(2); DOI: $10.31579 / 2693-2156 / 023$

Copyright: ( 2021 Sidi Mohamed Debbal. This is an open access article distributed under the Creative Commons Attribution License, which permits unrestricted use, distribution, and reproduction in any medium, provided the original work is properly cited.

\begin{abstract}
Phonocardiogram (PCG) signal is a particular approach to explore cardiac activity, to develop technics that may serve medical staff to diagnose several cardiac diseases. We took advantage of PCG signal that shows heart murmurs on its tracing dissimilar to other cardiac signals, to design an algorithm to study and classify heart murmurs. In this paper, the importance is given to the severity of murmurs to highlight its impact, since depending on its stage the patient could be in life-threatening point; therefore, the purpose of this paper is focused on three essential steps: according to the algorithm, extracting murmurs and classifying them to deferent stages then investigate the impact of severity on cardiac frequency through some parameters. The severity stage calculation was based on energy ratio (ER) which is recommended by recent studies as an effective factor, however, we succeed to validate that murmur energy (ME) is also a qualified feature to determine severity. But despite that murmur duration, it's an inefficient way to judge the cardiac severity, which is a very important indicator of the general health of the human body. This study is done on considering many patients and it reveals very interesting results.
\end{abstract}

Key words: severity; energy ratio; cardiac frequency; phonocardiogram signal; murmur

\section{Introduction}

Phonocardiogram signal is the graphical representation for the audible heart sounds commonly known as heartbeats; a normal representation is composed of two main sounds S1 and S2. The sound S1 corresponding to the beginning of the ventricular systole is due to the closure of the atrioventricular valves. This sound is composed of four internal components, two of which are the mitral component M1 (associated with mitral valve closure) and the tricuspid component T1 (associated with the closure of the tricuspid valve). The sound S2, which marks the end of the ventricular systole and signifies the beginning of the diastole, is composed of two main components: the aortic component A2 (corresponding to the closure of the aortic valve) and the pulmonary component P2 (corresponding to closure of the pulmonary valve) $[1,2,3]$.

Phonocardiogram (PCG) signal as a physiological signal can be affected by several types of pathologies that can occur on the graphical representation, one of the most common disease are murmurs, which are any sound in the heart region other than normal heart sounds; common causes include movement of blood through narrowed or stenosis heart valves and blood leaking through a valve that does not close properly. In many cases a murmur may be of the innocent or functional type, with no heart disease at all, so that it causes no trouble; this type is only sporadically present and in time may go away completely [4]. The following figure (Fig.1) represents the difference between normal heart sounds and PCG affected by murmurs. Also, it should be notice that:

- $\quad$ Systolic murmur: occurs during a heart muscle contraction. Systolic murmurs are divided into ejection murmurs (due to blood flow through a narrowed vessel or irregular valve) and regurgitant murmurs.

- Diastolic murmur: occurs during heart muscle relaxation between beats. Diastolic murmurs are due to anarrowing (stenosis) of the mitral or tricuspid valves, or regurgitation of the aortic or pulmonary valves $[\mathrm{A}]$. 

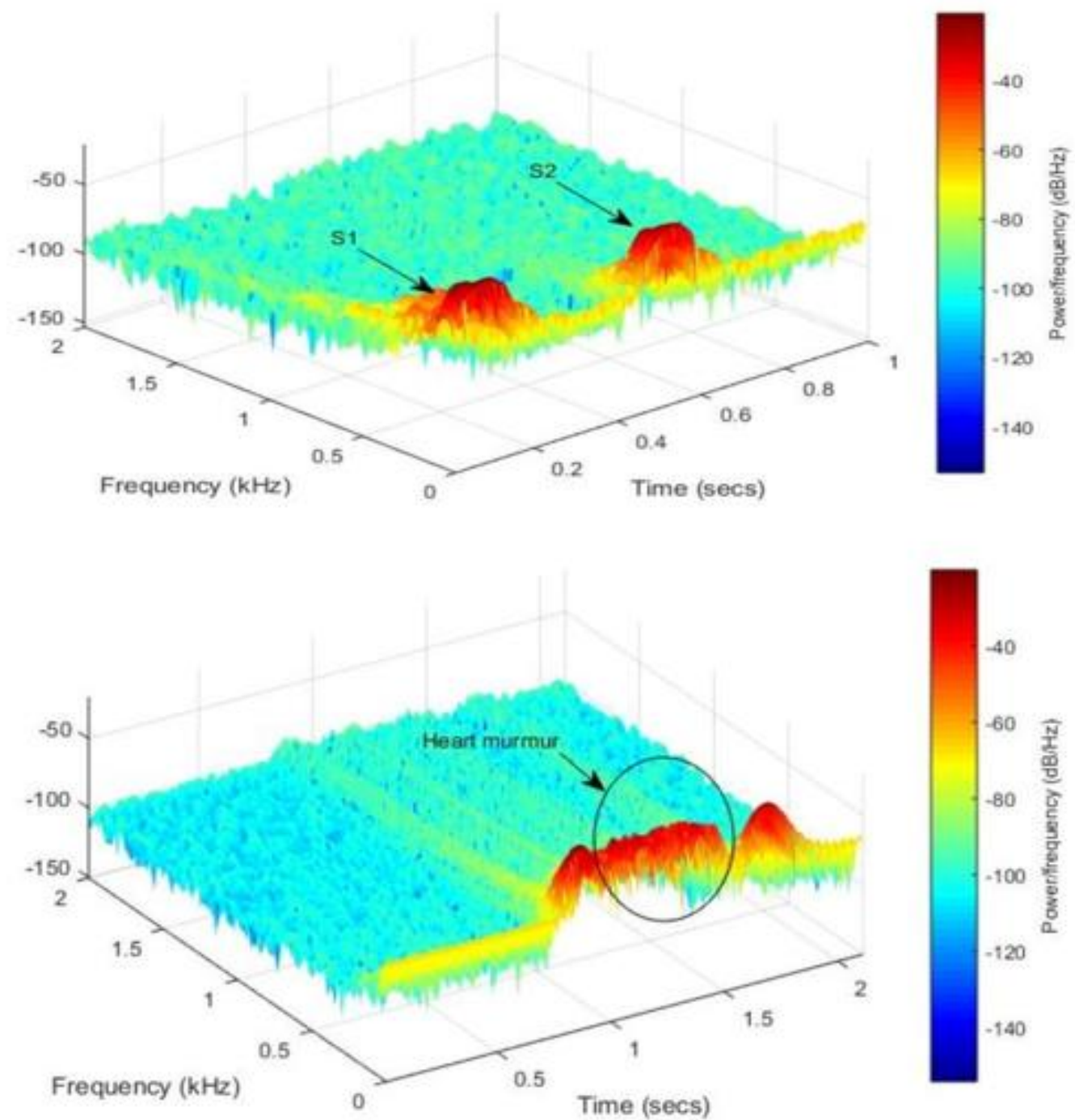

Figure 1. Difference between a normal PCG and a supervene murmur

\section{Materials and Methods}

The electrocardiogram signal (ECG) is a widely used technic when it comes to cardiac exploitation scientific research, but unfortunately, it remains limited and it's incapable to give all information about heart pathologies like heart murmurs, which do not appear in the ECG tracing. Hence the need to look for an alternative to characterize them has inspired us in the present work to use the phonocardiogram signal (PCG) approach to solve this problem. Huge importance is given in this paper to determine the severity of the murmurs and their impact on cardiac variability. An algorithm has been designed to extract features to better understand this phenomenon, this algorithm is founded on two essential steps:
a) Murmurs extraction
b) Severity calculation

\section{Murmurs extraction}

Murmurs extraction has been done on previous work [5,6] by calculating the average Shannon energy envelop to set the beginnings and ends of heart sounds and heart murmurs and isolate individual sounds (S1 or S2) and hold only murmurs. Since the aim of this work is not to explain the separation process we are passing it to the next step.

\section{Severity calculation}

Once murmurs are extracted, we classify them according to their severity. They are many techniques to calculate the severity [7] a used gradient of pressure and blood velocity nevertheless this technique shows some limitations because of its dependence on transvascular flow, in the same context the American Heart Association (AHA) and the American College of Cardiology (ACC) recommend using the valvular area to quantify the severity [8] but this parameter may tend to overestimate the severity and its need specific care during patient examination, D Kim et al reached to measure the duration of murmur at $300 \mathrm{~Hz}$ across a PCG time-frequency representation to define severity [9] but this technique still not give all about murmurs because chronology doesn't reflect the intensity of murmur that's why in the paper we try to proceed a method 
that explores the real severity of the murmur. The severity calculation process is built on energy calculation as an important factor to define the total presence of murmur on the cardiac cycle by comparing it to the energy of the other major sounds S1 and S2. Based on previous studies [10-12] that have been shown that energy ratio is a fair clue and useful argument for severity classification, energy ratio ER is given by the following equation:

$$
R E=\mathrm{E}_{\text {murmur }} /\left(\mathrm{E}_{S 1}+\mathrm{E}_{\text {murmur }}+\mathrm{E}_{S 2}\right)
$$

Where:

$\mathrm{E}_{\text {murmur: }}$ is the energy of murmur, $\mathrm{E}_{S I}$ : energy of the first heart sound $\mathrm{S} 1$, $\mathrm{E}_{S 2}$ : energy of the second heart sound S2. Murmurs are classified by multiplying RE by 100 to get a percentage in order that what is between:

- $1 \%<\mathrm{RE}<30 \%$ is considered Mild murmur.

- $\quad 30 \%<\mathrm{RE}<70 \%$ is considered Medium murmur.

- $70 \%<\mathrm{RE}<100 \%$ is considered Severe murmur.

Database of cardiac abnormalities of heart sounds was taken from [B,C]. The abbreviations of PCG signals used in this study and their sampling frequencies are given in Table 1.

\begin{tabular}{|l|l|l|}
\hline PCG signals & Abreviation & Sampling frequency (Hz) \\
\hline Aortic Stenosis & AS & 22050 \\
\hline Systolic Mitral Prolapse & SMP & 11025 \\
\hline Mitral Stenosis & MS & 8012 \\
\hline Aortic Regurgitation & AR & 8012 \\
\hline
\end{tabular}

Table 1. The abbreviations of PCG signals and their sampling frequencies

\section{Results and Discussion}

This study reveals very interesting results that can be arranged on three points: severity of murmur calculation and classifying them into degrees, studying the impact of this severity on the cardiac frequency, and the link between murmur duration over heart cycle and its severity. Table. 2 reflects the efficiency of using energy ratio $\mathrm{RE}$ as a procedure to define the stage of heart pathologies from mild and medium to severe pathology that needs to pay attention and have special care or an emergency medical intervention in some cases.

\begin{tabular}{|l|l|l|l|l|l|}
\hline subjects & ES & ER $\%$ & $\begin{array}{l}\text { Cycle duration } \\
\text { (second) }\end{array}$ & $\begin{array}{l}\text { Cardiac frequency } \\
\text { Fc (Hz) }\end{array}$ & severity \\
\hline 1 & 370 & 90 & 0.2521 & 3.93 & severe \\
\hline 2 & 266 & 89 & 0.2580 & 3.87 & severe \\
\hline 3 & 204 & 62 & 0.7863 & 1.2718 & medium \\
\hline 4 & 193.5 & 49.8 & 0.8722 & 1.1494 & medium \\
\hline 5 & 132.7 & 48.4 & 0.7863 & 1.2728 & medium \\
\hline 6 & 100.5 & 48.2 & 0.6562 & 1.5352 & medium \\
\hline 7 & 85 & 36.23 & 0.8688 & 1.1513 & medium \\
\hline 8 & 84 & 36.05 & 0.8677 & 1.1524 & mild \\
\hline 9 & 79 & 13.47 & 0.7875 & 1.2698 & mild \\
\hline 10 & 78 & 13.12 & 0.7760 & 1.2886 & mild \\
\hline 11 & 75 & 11.13 & 0.7627 & 1.3111 & mild \\
\hline 12 & 74 & 3.33 & 0.7475 & 1.3411 & \\
\hline
\end{tabular}

Table 2. Summary of deferent features calculated

The table. 2 shows the results beyond RE calculation for AS case and the same work was applied on the other pathologies mentioned above (table.1).

\section{Correlation between murmur's energy and energy ratio}

In the first place, we tried to find the correlation between murmur energy and the severity degree, to validate that murmur energy can be also an effective way of judging heart disease severity. Figures from 2 to 5 show a strong correlation between these two features (Murmur energy /severity).

Signals in this stage are arranging from 1 st degree to 5 th $/ 6$ th degree based on the energy ratio RE method.

The evolution of murmur energy points on graphs is very close to the increasing slope of optimization, which implies that a heavy murmur (high energy) is automatically a severe murmur with a proportional relation. 


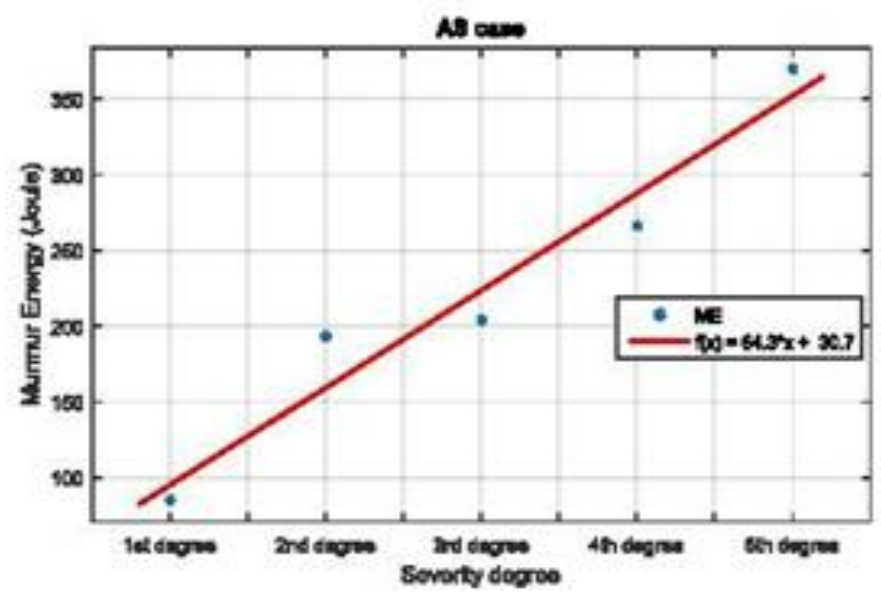

Figure 2. Correlation between murmur energy (ME) and severity degree (AS case)
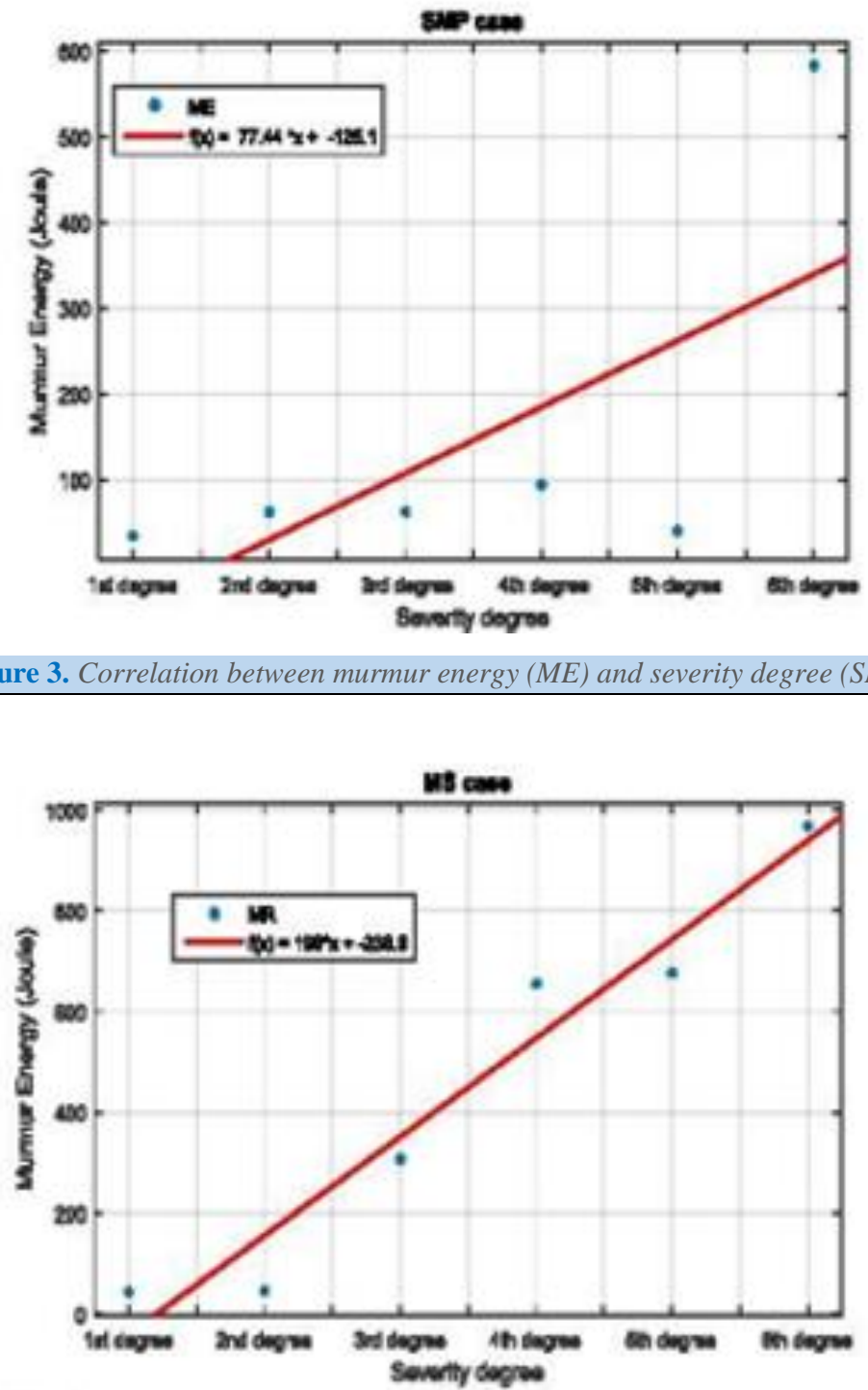

Figure 4. Correlation between murmur energy (ME) and severity degree (MS case) 


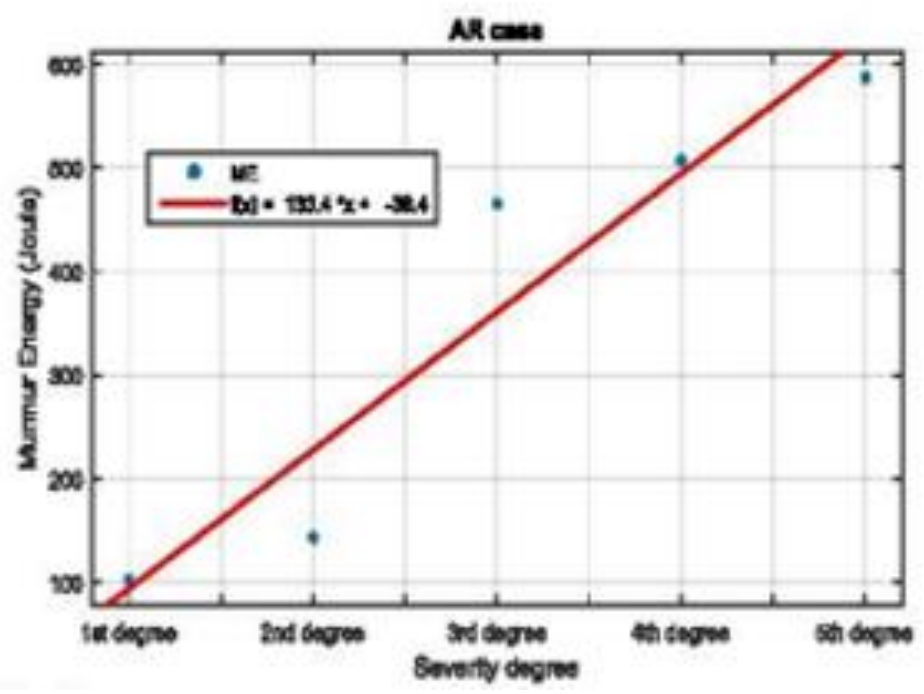

Figure 5. Correlation between murmur energy (ME) and severity degree (AR case)

Since we are taking advantage form the nature of the phonocardiogram signal that shows murmurs on its graphical tracing, some features have been extracted to figure out the impact of severity of these murmurs on cardiac frequency, but first we need to define the cardiac frequency which is the inverse of duration $\mathrm{T}$ between two successive peaks of the first sound $\mathrm{S} 1$ as given by the equation below:

$$
\mathrm{F}_{\mathrm{C}}=1 / \mathrm{T}
$$

It should be noticed that the cardiac frequency here is not the heart rate well known as Bpm (number of beats per minute), while the cardiac frequency is a parameter with the unit (Hertz).
For a healthy adult, at rest, the average heartbeat is $75 \mathrm{Bpm}$ and for this, the cardiac cycle time is $0.8 \mathrm{sec}$ and $\mathrm{Fc}=1.25 \mathrm{~Hz}$ (theoretically). The cardiac frequency for a normal person who presents no pathology has been calculated by our algorithm is around: $1.2475 \mathrm{~Hz}$ (the average value) and the findings in table 2 will be compared by this value $[13,14]$.

\section{The impact of severity on cardiac frequency}

To better understand these phenomena we extended the study to comprise the variation of the cardiac frequency over severity from $1 \mathrm{st}$ degree to 4 th degree as illustrated by the following figures.

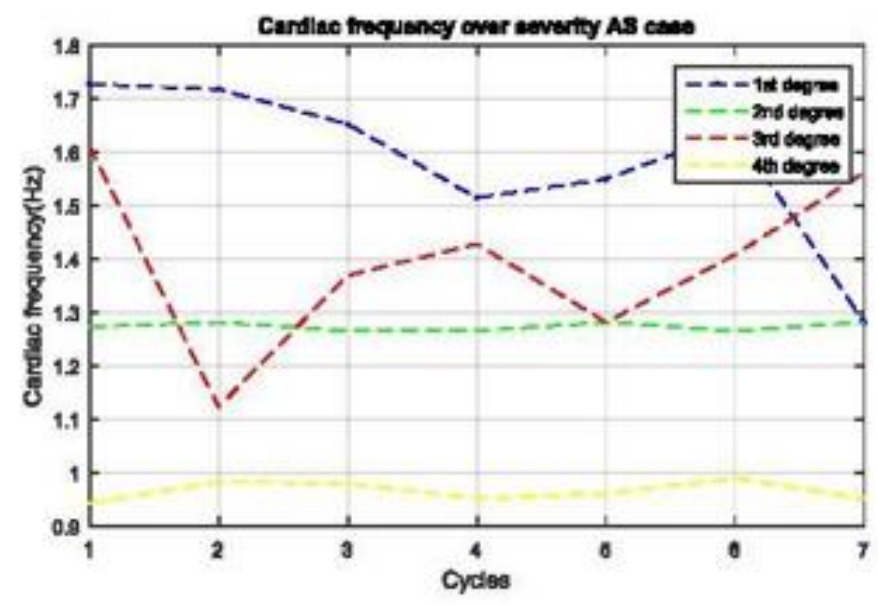




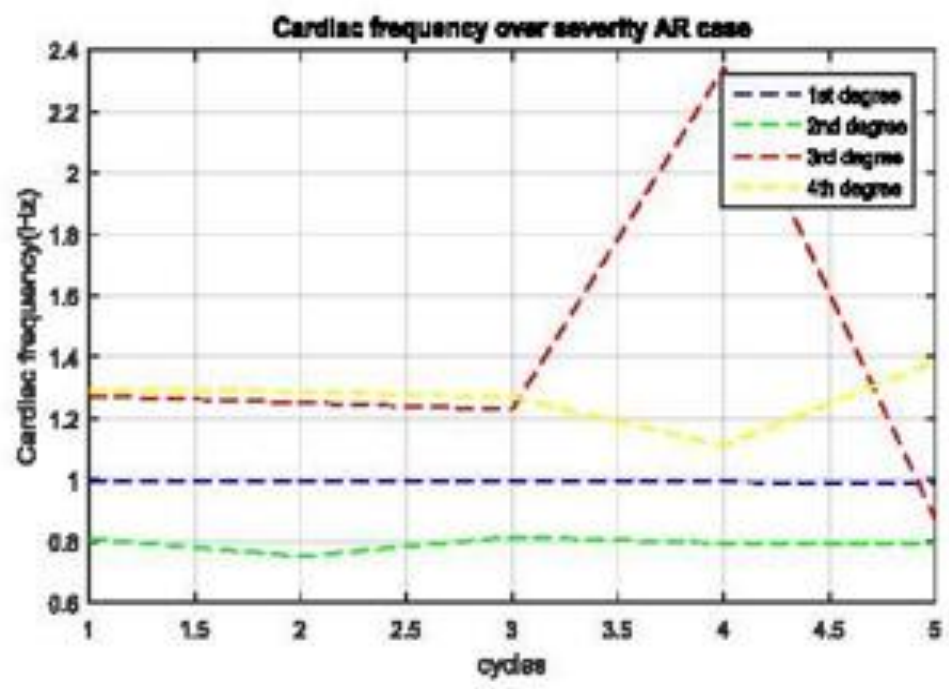

Figure 7. Cardiac frequency over severity degree (AR case)

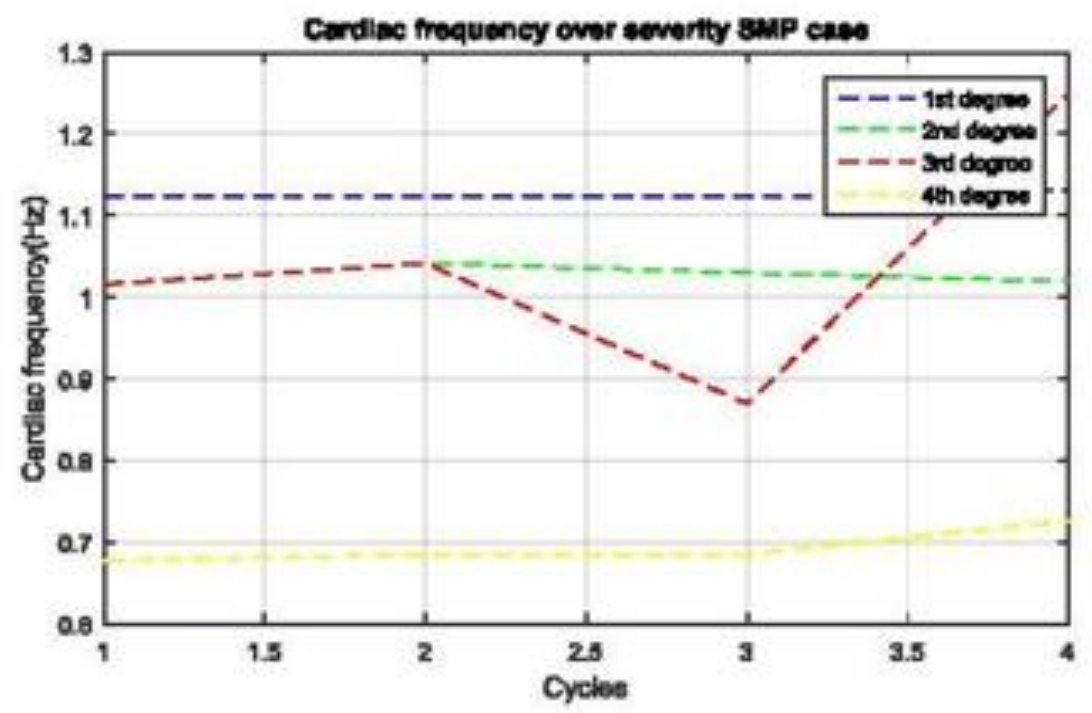

Figure 8. Cardiac frequency over severity degree (SMP case)

These figures reveal very interesting results and show that the severity not only affects the cardiac frequency in a general way because it's normally fluctuating around $1.2475 \mathrm{~Hz}$ for healthy persons (here's the $\mathrm{Fc}$ of all pathologies is deferent from this value) but also affecting this variability in such a way: according to the table. 2 cardiac frequency is hugely affected by murmur's severity where it jumps to highest values $(3.9 \mathrm{~Hz})$ for severe murmur, which means noticeable heartbeats and uncomfortable symptoms, according to the reference ${ }^{\mathrm{D}}$ heart becomes weak and needs to work harder to pump blood through the body, experts classify severe AS as a serious matter because it's related to the aortic valve damage and it could reach to a life-threatening point.

Besides figures from 6 to 8 present a high variation of cardiac frequency evolution for each pathology from 1 st to 4 th degree which means a remarkable variation that we quantified by $\Delta \mathbf{F}$ (deference between the average value of $\mathrm{Fc}_{1 s t}$ and $\mathrm{Fc}_{4 \text { th }}$ degree) presented by the following findings:

$$
\Delta \mathrm{F}_{\mathrm{AS}}=0.6175 \mathrm{~Hz}, \Delta \mathrm{F}_{\mathrm{AR}}=0.2775 \mathrm{~Hz}, \Delta \mathrm{F}_{\mathrm{SMP}}=0.4316 \mathrm{~Hz}
$$



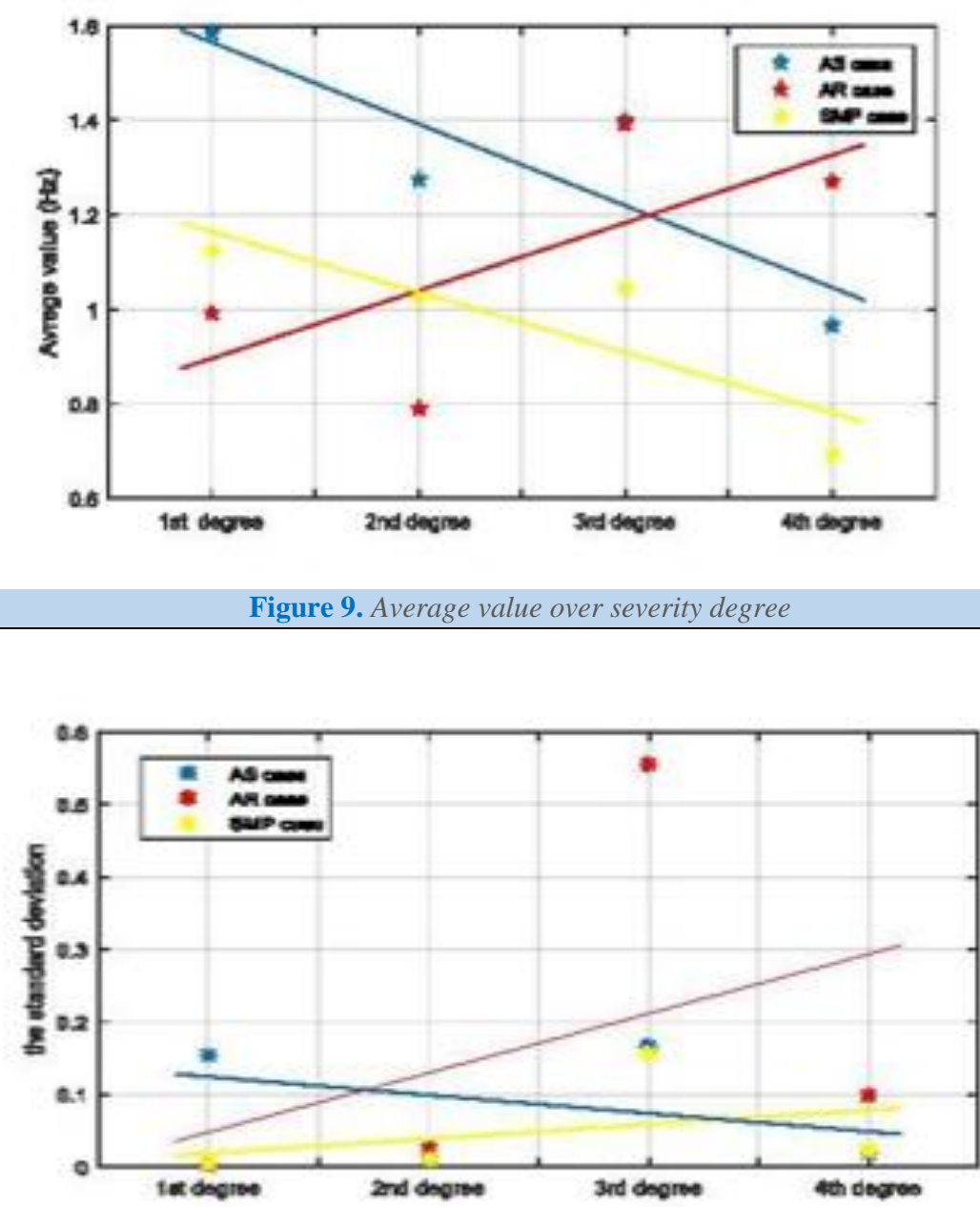

Figure 10. The standard deviation over severity degree

The previous figures Fig.9 and Fig.10 reflect the impact of severity by two important features, which are the standard deviation and the average value frequency over severity for each pathology.

At the first glance the average values of Fc (Fig.9) seem like a random distributed points cloud because it's limited in a small interval [0.8-1.6] $\mathrm{Hz}$, the optimization lines show the real tendency, whatever ascending or descending over severity it reveals on a very interesting result about severity impact.

Also according to Fig.10, the standard variation numbers over severity are attended to be convergent in an advanced stage of severity for AS case and more divergent for MS and SMP cases, which can be a highlight result for this study.

\section{Correlation between murmur's severity and duration}

The point behind the determination of this relation is to figure out if a long duration of a heart murmur means a severe stage of the pathology, nevertheless, the representation in Fig.11 shows aleatory fluctuations between murmur's duration and degree of severity of each PCG signal, ranging from 1st to 4th stage, the fluctuations did not take an increasing order which answers the hypothesis mentioned above. We recommend that the duration of a murmur is an insufficient criterion for determining the degree of severity. 


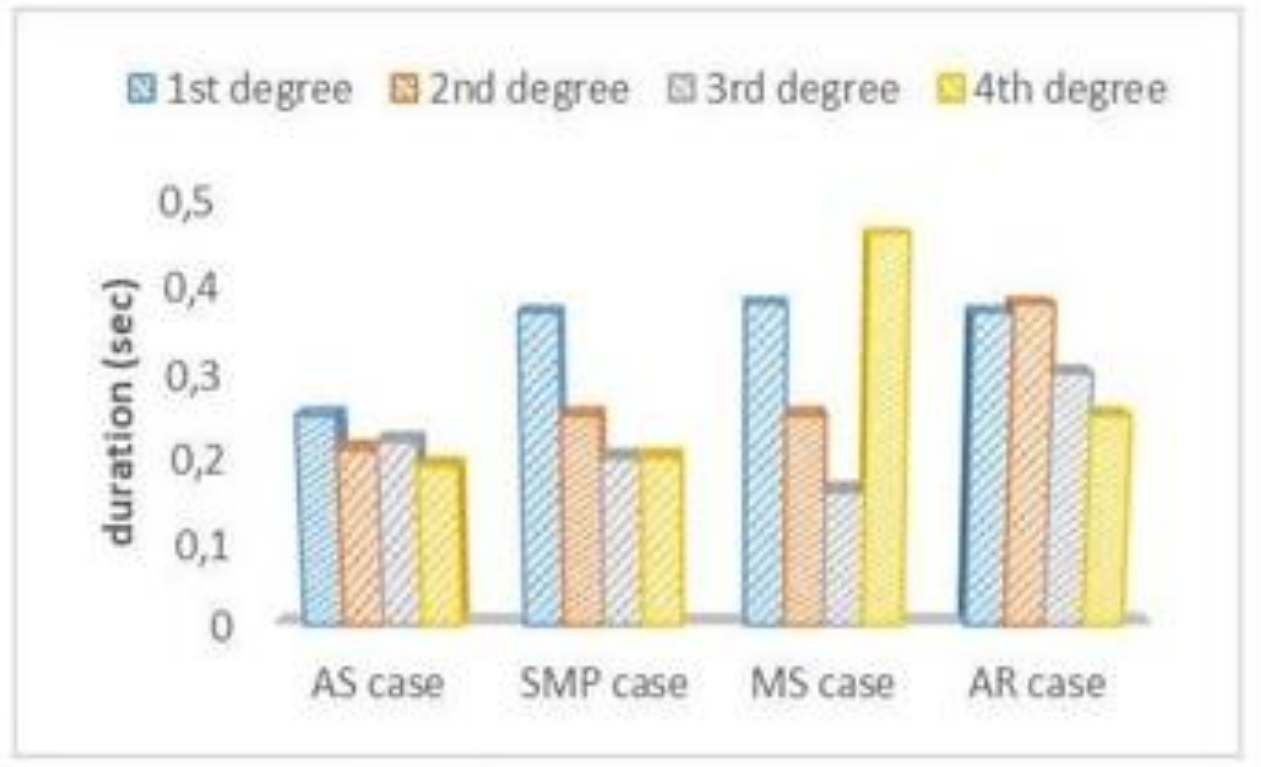

Figure 11. Duration over severity degree

\section{Conclusion}

Heart murmurs are a serious health matter in the entire world and phonocardiogram signal is a particular approach to extract as much possible information about murmurs. In this study, we tried to focus on severity and their impact on cardiac frequency.

Energy ratio shows its efficiency as an important process to calculate the severity stage of a heart murmur and classify them according to ER to mild, medium, and severe murmurs. Also, murmur's energy (MR) has been demonstrating a strong correlation with severity, it can be also a qualified feature to classify heart murmurs severity. The study reveals on very interesting result concerning the impact of severity on the cardiac frequency where it's hugely affected by severe murmurs, features like $\Delta \mathrm{F}$, the average value, and the standard deviation highlight this result. As well and according to this study, we have been confirmed that murmur duration, it cannot be an adequate criterion to judge the severity and it should be always checked by another method. All these results obtained can be further improved and reinforced if this statistical study can be done on a larger number of pathologies.

\section{Acknowledgments}

The authors would like to thank the Directorate-General of Scientific Research and Technological Development (Direction Générale de la Recherche Scientifique et du Développement Technologique, DGRSDT, URL: www.dgrsdt.dz, Algeria) for the financial assistance towards this research.

\section{References:}

1. A.Donant, J.Bourneuf. (1981). Nouveau Larousse Médicale, Edition Larousse, 1981.

2. M.S. Obaidat Phonocardiogram signal analysis: techniques and performance comparison. Journal of Medical Engineering \& technologie, vol 17, No 6 (November-December 1993), 221-227.

3. C. Ahlstrom. (2006). Processing of the Phonocardiographic Signal -Methods for the Intelligent Stethoscope, Department of Biomedical Engineering Linköpings universitet, SE-58185 Linköping, Sweden, 2006.

4. Dorland's Medical Dictionary 2007 edition elsiver
5. L. Hamza Cherif, et al. (2008). Segmentation of heart sounds and heart murmurs; Journal of Mechanics in Medicine and Biology Vol. 8, No. 4 (2008) 549-559.

6. J. P. Ramos et al; Intelligent phonocardiogram analysis representation tool, Department of Informatics Engineering, University of Coimbra, Polo II, Coimbra, Portugal, BIOSTEC 2011.

7. Raj.R. Makkar,et al. (2019). Association Between Transcatheter Aortic Valve Replacement for Bicuspid vs Tricuspid Aortic Stenosis and Mortality or Stroke ; JAMA. 2019 Jun 11; 321(22): 2193-2202.

8. Ontario Health (Quality). (2020). Transcatheter Aortic Valve Implantation in Patients with Severe, Symptomatic Aortic Valve Stenosis at Intermediate Surgical Risk: A Health Technology Assessment; Ont Health Technol Assess Ser. 2020; 20(2): 1121.

9. Kim D1, Tavel ME. (2003). Assessment of severity of aortic stenosis through time-frequency analysis of murmur; $\mathrm{J}$ american college of Chest physicians. 2003 Nov; 124(5):1638-1644.

10. Sumair Aziz et al. (2020). Phonocardiogram Signal Processing for Automatic Diagnosis of Congenital Heart Disorders through Fusion of Temporal and Cepstral Features; Sensors (Basel). 2020 Jul; 20(13): 3790.

11. M. Omari Taher. (2009). Study of the degree of pathological severity of aortic stenosis; Magister thesis in biomedical electronics, University of Tlemcen, June 2009.

12. F.Meziani, (2013). Analysis of the degree of pathological severity of phono cardiogram signals (PCGs) by application of wavelet transforms, doctoral thesis in biomedical, University of Tlemcen, June 2013.

13. Tom Kenny. (2015). The Nuts and Bolts of Implantable Device Therapy: Pacemakers, John Wiley \& Sons, 20 janv. 2015 - 2336 pages.

14. F. Mokeddem, et al. (2020). Study of murmurs and their impact on the heart Variability Int. J. Medical Engineering and Informatics, Vol. 12, No. 3, 2020.

A. https://stanfordhealthcare.org/medicalconditions/blood-heart-circulation/heartmurmurs/types.html 
B. American college of cardiology.[http://www.Egeneral medical.com].

C. http://www.cardiosource.com/heart sounds.
D. https://newheartvalve.com/understand-aorticstenosis/dangers-severe-aortic-stenosis/
Ready to submit your research? Choose Auctores and benefit from:

* fast, convenient online submission

* rigorous peer review by experienced research in your field

* rapid publication on acceptance

* authors retain copyrights

* unique DOI for all articles

* immediate, unrestricted online access

At Auctores, research is always in progress.

Learn more www.auctoresonline.org/journals/journal-of-thoracicdisease-and-cardiothoracic-surgery- 